PROCEEDINGS OF THE

AMERICAN MATHEMATICAL SOCIETY

Volume 136, Number 11, November 2008, Pages 3861-3867

S 0002-9939(08)09475-6

Article electronically published on June 3, 2008

\title{
ON THE NÉRON-SEVERI GROUP OF SURFACES WITH MANY LINES
}

\author{
SAMUEL BOISSIÈRE AND ALESSANDRA SARTI
}

(Communicated by Ted Chinburg)

\begin{abstract}
For a binary quartic form $\phi$ without multiple factors, we classify the quartic $K 3$ surfaces $\phi(x, y)=\phi(z, t)$ whose Néron-Severi group is (rationally) generated by lines. For generic binary forms $\phi, \psi$ of prime degree without multiple factors, we prove that the Néron-Severi group of the surface $\phi(x, y)=\psi(z, t)$ is rationally generated by lines.
\end{abstract}

\section{INTRODUCTION}

The study of the Néron-Severi group $\mathrm{NS}(S)$ of a given surface $S$ is interesting for understanding its geometry, but it is not an easy task in general. A first step is to compute its Picard number $\rho(S):=\operatorname{rk} \operatorname{NS}(S)$. A second step is to give a family of generators of $\mathrm{NS}(S)$ over $\mathbb{Z}$. To this purpose, it is very useful to first find a nice family of generators of $\mathrm{NS}(S) \otimes_{\mathbb{Z}} \mathbb{Q}$. If one already knows the value of the determinant of $\operatorname{NS}(S)$, this can help in deducing a family of generators. If not, the study of the rational generators gives nontrivial information for the value of the discriminant.

Let $\phi$ be a binary quartic form without multiple factors. After a suitable linear change of coordinates, we may assume that $\phi$ is of the form

$$
\phi(x, y)=y x(y-x)(y-\lambda x)
$$

for $\lambda \in \mathbb{C} \backslash\{0,1\}$. Naturally associated to $\phi$ are the $K 3$ surface $S_{\phi}: \phi(x, y)=\phi(z, t)$ and the elliptic curve $E_{\phi}: t^{2}=\phi(1, y)$.

Remark 1.1. Observe that if $\phi, \phi^{\prime}$ are the forms corresponding to $\lambda, \lambda^{\prime}$ and $\lambda^{\prime}$ is one of the values $\lambda, \frac{1}{\lambda}, 1-\lambda, \frac{1}{1-\lambda}, \frac{\lambda}{\lambda-1}, \frac{\lambda-1}{\lambda}$, then there is a linear isomorphism $S_{\phi} \cong S_{\phi^{\prime}}$.

The interplay between the geometry of the $K 3$ surface $S_{\phi}$ and the arithmetic of the elliptic curve $E_{\phi}$ has been studied by many authors. Of particular interest is the link between the value of the Picard number $\rho\left(S_{\phi}\right)$ and the existence of a complex multiplication on $E_{\phi}$. The following result is classical (see Kuw95 and the references therein):

$$
\rho\left(S_{\phi}\right)= \begin{cases}20 & \text { if } E_{\phi} \text { has a complex multiplication, } \\ 19 & \text { otherwise }\end{cases}
$$

Received by the editors January 22, 2007, and, in revised form, March 29, 2007, and October 9, 2007.

2000 Mathematics Subject Classification. Primary 14J28.

Key words and phrases. Néron-Severi group, Picard number, lines on surfaces.

(C)2008 American Mathematical Society 3861

Reverts to public domain 28 years from publication 
We pursue the study by giving numerical conditions for the Néron-Severi group of $S_{\phi}$ to be rationally generated by lines:

Notation-Definition. Let $S \subset \mathbb{P}_{\mathbb{C}}^{3}$ be a smooth surface of degree $d \geq 3$. If $L$ is a line contained in $S$, by the genus formula the self-intersection of $L$ in $S$ is $L^{2}=-d+2$, so the class of $L$ in $\operatorname{NS}(S)$ is not a torsion class. We denote by $\operatorname{LC}(S)$ the sublattice of the torsion-free part of $\operatorname{NS}(S)$ generated by the classes of the lines contained in $S$. For a generic surface $S$, it is well known that $\operatorname{LC}(S)=0$. If not, these classes are natural candidates as generators of $\mathrm{NS}(S)$ and we say that $\mathrm{NS}(S)$ is rationally generated by lines if $\operatorname{rk} \operatorname{LC}(S)=\rho(S)$, that is, $\operatorname{LC}(S) \otimes_{\mathbb{Z}} \mathbb{Q}=\operatorname{NS}(S) \otimes_{\mathbb{Z}} \mathbb{Q}$.

The most famous examples of surfaces whose Néron-Severi group is rationally generated by lines are certain Fermat surfaces (see [Shi81]). The surfaces we study here are a natural generalization of them. We prove (\$2):

Theorem 1.2. The Néron-Severi group of $S_{\phi}$ is rationally generated by lines exactly in the following cases:

(1) $\lambda \notin \overline{\mathbb{Q}}$;

(2) $\lambda \in\left\{-1,2, \frac{1}{2}, \frac{1+\mathrm{i} \sqrt{3}}{2}, \frac{1-\mathrm{i} \sqrt{3}}{2}\right\}$;

(3) $\lambda \in \overline{\mathbb{Q}} \backslash\left\{-1,2, \frac{1}{2}, \frac{1+\mathrm{i} \sqrt{3}}{2}, \frac{1-\mathrm{i} \sqrt{3}}{2}\right\}$ and $\rho\left(S_{\phi}\right)=19$.

Looking now for a set of generators of the Néron-Severi group, we prove ( $\sqrt{3})$ :

Theorem 1.3. The Néron-Severi group of $S_{\phi}$ is generated by lines only in case (2).

Generalizing the construction, one can consider two binary forms $\phi, \psi$ of degree $d$ without multiple factors and the associated surface $S_{\phi, \psi}^{d}: \phi(x, y)=\psi(z, t)$. One can prove that $\rho\left(S_{\phi, \psi}^{d}\right) \geq(d-1)^{2}+1$ with equality for $d$ prime and $\phi, \psi$ generic (see Sas68). We prove (44):

Theorem 1.4. For $d$ prime and $\phi, \psi$ generic, the Néron-Severi group of $S_{\phi, \psi}^{d}$ is rationally generated by lines.

In Theorem 1.2 we do not consider the quartics $S_{\phi, \psi}^{4}$ for $\phi \neq \psi$ since, although $\rho\left(S_{\phi, \psi}^{4}\right)=18$ (see again [Kuw95]), Proposition 4.1 below says that their 16 lines generate an intersection matrix of rank 10, so such surfaces do not enter in our context.

\section{Proof of Theorem 1.2}

The result follows from the following proposition:

Proposition 2.1. If $\lambda \in\left\{-1,2, \frac{1}{2}, \frac{1+\mathrm{i} \sqrt{3}}{2}, \frac{1-\mathrm{i} \sqrt{3}}{2}\right\}$, then $\operatorname{rk} \operatorname{LC}\left(S_{\phi}\right)=20$; otherwise $\operatorname{rk~LC}\left(S_{\phi}\right)=19$.

Proof of Theorem 1.2, Assuming Proposition 2.1, we prove Theorem 1.2, The key argument is that if $E_{\phi}$ has a complex multiplication, then its $j$-invariant is algebraic over $\overline{\mathbb{Q}}$ (see [Sil94]). Since $j\left(E_{\phi}\right)=\frac{256\left(1-\lambda+\lambda^{2}\right)^{3}}{\lambda^{2}(\lambda-1)^{2}}, j\left(E_{\phi}\right) \in \overline{\mathbb{Q}}$ if and only if $\lambda \in \overline{\mathbb{Q}}$. Then:

If $\lambda \notin \overline{\mathbb{Q}}, E_{\phi}$ has no complex multiplication, so $\rho\left(S_{\phi}\right)=19$ and by Proposition 2.1. $\operatorname{rk} \operatorname{LC}\left(S_{\phi}\right)=19$. This proves (1).

If $\lambda \in\left\{-1,2, \frac{1}{2}, \frac{1+\mathrm{i} \sqrt{3}}{2}, \frac{1-\mathrm{i} \sqrt{3}}{2}\right\}$, by Proposition 2.1 we have $\operatorname{rk} \operatorname{LC}\left(S_{\phi}\right)=20$, so $\rho\left(S_{\phi}\right)=20$. This proves $(2)$. 
If $\lambda \in \overline{\mathbb{Q}} \backslash\left\{-1,2, \frac{1}{2}, \frac{1+\mathrm{i} \sqrt{3}}{2}, \frac{1-\mathrm{i} \sqrt{3}}{2}\right\}$, then $\rho\left(S_{\phi}\right) \in\{19,20\}$ and $\operatorname{rk} \mathrm{LC}\left(S_{\phi}\right)=19$. This gives (3).

Remark 2.2. In case (3) of Theorem 1.2, one cannot be more precise since:

- When $j\left(E_{\phi}\right) \in \overline{\mathbb{Q}}$ (so $\left.\lambda \in \overline{\mathbb{Q}}\right)$, it is not clear whether $E_{\phi}$ admits a complex multiplication or not.

- There is a dense and numerable set of $\lambda \in \overline{\mathbb{Q}}$ such that $\rho\left(S_{\phi}\right)=20$ (see Ogu $)$.

Proof of Proposition 2.1. The description of the lines on $S_{\phi}$ comes from Segre Seg47. We follow the presentation given in BS07.

Case 1. If $\lambda \notin\left\{-1,2, \frac{1}{2}, \frac{1+\mathrm{i} \sqrt{3}}{2}, \frac{1-\mathrm{i} \sqrt{3}}{2}\right\}$, the group of automorphisms of $\mathbb{P}_{\mathbb{C}}^{1}$ permuting the set $\{\infty, 0,1, \lambda\}$ is the dihedral group $D_{2}=\left\{i d, s_{1}, s_{2}, s_{1} s_{2}\right\}$ and the surface $S_{\phi}$ contains exactly the following 32 lines:

$$
\begin{array}{r}
\ell_{z}(u, v):\left\{\begin{array}{l}
v x=u y \\
v=u z
\end{array} \quad \ell_{i d}(p):\left\{\begin{array}{l}
x=p z \\
y=p t
\end{array} \quad \ell_{s_{1}}(p):\left\{\begin{array}{l}
x=p z-p t \\
y=\lambda p z-p t
\end{array}\right.\right.\right. \\
u, v \in\{\infty, 0,1, \lambda\} \quad p \in\{1,-1, \mathrm{i},-\mathrm{i}\} \\
\ell_{s_{2}}(p):\left\{\begin{array}{l}
x=p t \\
y=\lambda p z
\end{array} \quad \ell_{s_{1} s_{2}}(p):\left\{\begin{array}{l}
x=-\lambda p z+p t \\
y=-\lambda p z+\lambda p t
\end{array}\right.\right. \\
p \in\left\{\frac{1}{\sqrt{\lambda}}, \frac{-1}{\sqrt{\lambda}}, \frac{\mathrm{i}}{\sqrt{\lambda}}, \frac{-1}{\sqrt{\lambda}}\right\}
\end{array}
$$

The intersection matrix of these 32 lines is easy to compute (we do not reproduce it here) and is independent of $\lambda$. One finds that its rank is $19, \operatorname{so} \operatorname{rk} \operatorname{LC}\left(S_{\phi}\right)=19$.

Case 2. If $\lambda \in\left\{-1,2, \frac{1}{2}\right\}$, the surfaces are isomorphic to each other by Remark 1.1. The group of automorphisms is the dihedral group $D_{4}=\left\langle D_{2}, r\right\rangle$. The surface $S_{\phi}$ contains exactly 48 lines: the 32 preceding ones and 16 other lines. For $\lambda=-1$ for example, these lines are:

$$
\begin{aligned}
& \ell_{r}(p):\left\{\begin{array}{l}
x=p z+p t \\
y=-p z+p t
\end{array} \quad \ell_{r^{-1}}(p):\left\{\begin{array}{l}
x=-p z+p t \\
y=-p z-p t
\end{array} \quad p \in\left\{\frac{1+\mathrm{i}}{2}, \frac{1-\mathrm{i}}{2}, \frac{-1+\mathrm{i}}{2}, \frac{-1-\mathrm{i}}{2}\right\}\right.\right. \\
& \ell_{r s_{1}}(p):\left\{\begin{array}{l}
x=p t \\
y=p z
\end{array} \quad \ell_{s_{1} r}(p):\left\{\begin{array}{l}
x=-p z \\
y=p t
\end{array} \quad p \in\left\{\frac{1+\mathrm{i}}{\sqrt{2}}, \frac{1-\mathrm{i}}{\sqrt{2}}, \frac{-1+\mathrm{i}}{\sqrt{2}}, \frac{-1-\mathrm{i}}{\sqrt{2}}\right\}\right.\right.
\end{aligned}
$$

The rank of the intersection matrix of the 48 lines is $\operatorname{rk} \operatorname{LC}\left(S_{\phi}\right)=20$.

Case 3. If $\lambda \in\left\{\frac{1+\mathrm{i} \sqrt{3}}{2}, \frac{1-\mathrm{i} \sqrt{3}}{2}\right\}$, the surfaces are isomorphic to each other by Remark 1.1. The group of automorphisms is the tetrahedral group $T=\langle r, s\rangle$. The 
surface $S_{\phi}$ contains exactly the following 64 lines:

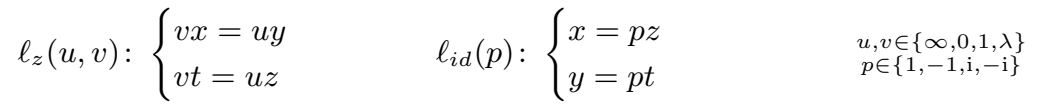

$$
\begin{aligned}
& \ell_{r}(p):\left\{\begin{array}{l}
x=p z \\
y=p z+\lambda^{2} p t
\end{array} \quad \ell_{r^{2}}(p):\left\{\begin{array}{l}
x=p z \\
y=\lambda p z-\lambda p t
\end{array}\right.\right. \\
& \ell_{s}(p):\left\{\begin{array}{l}
x=p t \\
y=\lambda p z
\end{array} \quad \ell_{r s}(p):\left\{\begin{array}{l}
x=p t \\
y=-p z+p t
\end{array} \quad \ell_{r s r}(p):\left\{\begin{array}{l}
x=p z+\lambda^{2} p t \\
y=\lambda^{2} p t
\end{array}\right.\right.\right. \\
& \ell_{r^{2} s}(p):\left\{\begin{array}{l}
x=p t \\
y=-\lambda^{2} p z+\lambda p t
\end{array} \quad \ell_{s r}(p):\left\{\begin{array}{l}
x=p z+\lambda^{2} p t \\
y=\lambda p z
\end{array} \quad p \in\{\lambda,-\lambda, \mathrm{i} \lambda,-\mathrm{i} \lambda\}\right\}\right.
\end{aligned}
$$

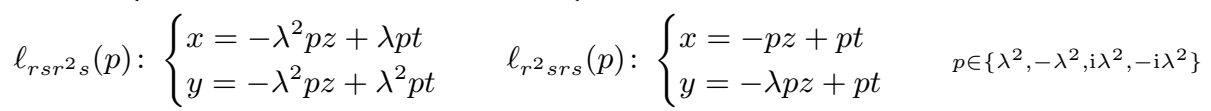

$$
\begin{aligned}
& \ell_{s r s}(p):\left\{\begin{array}{l}
x=-p z+p t \\
y=\lambda p t
\end{array} \quad \ell_{r s r s}(p):\left\{\begin{array}{l}
x=-p z+p t \\
y=-p z
\end{array}\right.\right.
\end{aligned}
$$

The rank of the intersection matrix of the 64 lines is $\operatorname{rk} \mathrm{LC}\left(S_{\phi}\right)=20$.

\section{Proof of Theorem 1.3}

As we explained in the Introduction, once one has found a nice family of rational generators of the Néron-Severi group, the next task is to get information on divisible classes. We call a divisor $\Lambda=\sum_{i=1}^{n} \alpha_{i} L_{i} \in \operatorname{NS}(S) 2^{m}$-divisible if the class of $\Lambda$ in $\operatorname{NS}(S)$ is divisible by $2^{m}$; for $m=1$ we say also that the lines in $\Lambda$ form an even set.

\section{Proof of Theorem 1.3.}

Cases (1) and (3). For $\lambda \notin\left\{-1,2, \frac{1}{2}, \frac{1+\mathrm{i} \sqrt{3}}{2}, \frac{1-\mathrm{i} \sqrt{3}}{2}\right\}$, with the help of a computer program we obtain that the best choice of a family of 19 lines among the 32 generating rationally the Néron-Severi group gives a determinant of value $2^{9}$. Denoting this lattice by $M$ and its dual by $M^{\vee}$, the discriminant group is

$$
M^{\vee} / M=\left(\mathbb{Z}_{2}\right)^{\oplus 2} \oplus\left(\mathbb{Z}_{4}\right)^{\oplus 2} \oplus \mathbb{Z}_{8}
$$

hence we can have only $2^{m}$-divisible classes for $m=1,2,3$. Denote by $\left(M^{\vee} / M\right)_{2}$ the part of the discriminant group generated by the 2-torsion classes. We have $\left(M^{\vee} / M\right)_{2}=\left(\mathbb{Z}_{2}\right)^{\oplus 5}$; hence $\operatorname{rank}\left(M^{\vee} / M\right)_{2}=5$. However, denoting by $T$ the transcendental lattice of $S_{\phi},\left(\mathrm{NS}\left(S_{\phi}\right)^{\vee} / \mathrm{NS}\left(S_{\phi}\right)\right)_{2} \cong\left(T^{\vee} / T\right)_{2}$ has rank at most the rank of $T$, which is three. This shows that $M \subsetneq \mathrm{NS}\left(S_{\phi}\right)$ and that there are at least two even sets of lines in the Néron-Severi group. In particular there is no set of 19 lines generating $\mathrm{NS}\left(S_{\phi}\right)$.

Case (2) for $\lambda \in\left\{-1,2, \frac{1}{2}\right\}$. By Remark [1.1, the surfaces $S_{\phi}$ are isomorphic to each other. The best choice of a family of 20 lines among 48 gives a determinant of value $-2^{6}$. Observe that a suitable permutation of the zeros of $x^{4}-y^{4}$ in $\mathbb{P}_{\mathbb{C}}^{1}$ gives a cross-ratio equal to -1 , so our surfaces are isomorphic to the Fermat quartic. It is then well known that $\operatorname{det} \operatorname{NS}\left(S_{\phi}\right)=-64$, so the lines generate the Néron-Severi group. 
Case (2) for $\lambda \in\left\{\frac{1+\mathrm{i} \sqrt{3}}{2}, \frac{1-\mathrm{i} \sqrt{3}}{2}\right\}$. A computer program shows that the best choice of a family of 20 lines among the 64 contained in the surface, generating rationally the Néron-Severi group, gives a determinant of value $-2^{4} \cdot 3$. We show in Appendix B that $\operatorname{det} \operatorname{NS}\left(S_{\phi}\right)=-48$, so the lines generate the Néron-Severi group.

\section{Proof of Theorem 1.4}

Since $\rho\left(S_{\phi, \psi}^{d}\right)=(d-1)^{2}+1$ for $d$ prime and $\phi, \psi$ generic, Theorem 1.4 follows from the following result.

Proposition 4.1. $\operatorname{rk} \mathrm{LC}\left(S_{\phi, \psi}^{d}\right)=(d-1)^{2}+1$.

Proof of Proposition 4.1. We set $S:=S_{\phi, \psi}^{d}$. Let $L$ be the line $z=t=0$ and $L^{\prime}$ be the line $x=y=0$. The intersection $S \cap L$ is the set of zeros of $\phi$, whereas $S \cap L^{\prime}$ is the set of zeros of $\psi$. If $p \in L$ is a zero of $\phi$ and $q \in L^{\prime}$ a zero of $\psi$, the line $L_{p, q}$ joining $p$ and $q$ is contained in $S$; this gives a family of $d^{2}$ lines contained in $S$. The intersection matrix of this family is given by $L^{2}=-d+2$ and $L \cdot L^{\prime}=1$ if $L$ and $L^{\prime}$ intersect, 0 otherwise. Note that

$$
\left(L_{p, q} \cap L_{p^{\prime}, q^{\prime}} \neq \emptyset\right) \Longleftrightarrow\left(p=p^{\prime} \text { or } q=q^{\prime}\right) .
$$

This implies that after ordering the lines correctly, the intersection matrix is the matrix $M_{d}:=K_{-d+2,1,1,0}^{d}$ (see the notation in Appendix A). Remark A.5 gives $\operatorname{rkLC}(S)=\operatorname{rk} M_{d}=(d-1)^{2}+1$.

\section{Appendix A. Some linear ALgebra}

Let $a, b, c, d, \ldots$ denote indeterminates. For $d \geq 2$, let $J_{a, b}^{d}$ be the $(d, d)$-matrix defined by

$$
J_{a, b}^{d}:=\left(\begin{array}{ccc}
a & & b \\
& \ddots & \\
b & & a
\end{array}\right)=b \cdot(1)+(a-b) \cdot I_{d}
$$

where $I_{d}$ denotes the identity $(d, d)$-matrix. The following lemma is clear.

Lemma A.1. The following identities hold:

$$
\begin{aligned}
J_{a, b}^{d}+J_{a^{\prime}, b^{\prime}}^{d} & =J_{a+a^{\prime}, b+b^{\prime}}^{d} \\
J_{a, b}^{d} \cdot J_{a^{\prime}, b^{\prime}}^{d} & =J_{a a^{\prime}+(d-1) b b^{\prime}, a b^{\prime}+a^{\prime} b+(d-2) b b^{\prime}}^{d}
\end{aligned}
$$

Now let $K_{a, b, c, d}^{d}$ be the $\left(d^{2}, d^{2}\right)$-matrix defined as the following $(d, d)$-blocks of $(d, d)$-matrices:

$$
K_{a, b, c, d}^{d}:=\left(\begin{array}{ccc}
J_{a, b}^{d} & & J_{c, d}^{d} \\
& \ddots & \\
J_{c, d}^{d} & & J_{a, b}^{d}
\end{array}\right) .
$$

The following lemma follows easily from Lemma A.1. 
Lemma A.2. The following identity holds:

$$
K_{a, b, c, d}^{d} \cdot K_{a^{\prime}, b^{\prime}, c^{\prime}, d^{\prime}}^{d}=K_{\alpha, \beta, \gamma, \delta}^{d},
$$

where

$$
\begin{aligned}
& \alpha=a a^{\prime}+(d-1)\left(b b^{\prime}+c c^{\prime}\right)+(d-1)^{2} d d^{\prime} ; \\
& \beta=a b^{\prime}+a^{\prime} b+(d-1)\left(c d^{\prime}+c^{\prime} d\right)+(d-2) b b^{\prime}+(d-1)(d-2) d d^{\prime} ; \\
& \gamma=a c^{\prime}+a^{\prime} c+(d-1)\left(b d^{\prime}+b^{\prime} d\right)+(d-2) c c^{\prime}+(d-1)(d-2) d d^{\prime} ; \\
& \delta=a d^{\prime}+a^{\prime} d+b c^{\prime}+b^{\prime} c+(d-2)\left(c d^{\prime}+c^{\prime} d+b d^{\prime}+b^{\prime} d\right)+(d-2)^{2} d d^{\prime} .
\end{aligned}
$$

Set $K_{d}:=K_{1,1,1,0}^{d}$. Its minimal polynomial $\mu_{K_{d}}(t)$ is given by:

Lemma A.3. $\mu_{K_{d}}(t)=(t-(d-1)) \cdot(t-(2 d-1)) \cdot(t+1)$.

Proof. Note that

$$
\begin{aligned}
K_{d}-(d-1) I_{d} & =K_{-d+2,1,1,0}^{d} ; \\
K_{d}-(2 d-1) I_{d} & =K_{-2 d+2,1,1,0}^{d} ; \\
K_{d}+I_{d} & =K_{2,1,1,0}^{d} .
\end{aligned}
$$

Applying Lemma A.2 one gets

$$
\begin{aligned}
K_{-d+2,1,1,0}^{d} \cdot K_{-2 d+2,1,1,0}^{d} & =K_{2(d-1)^{2},-2 d+2,-2 d+2,2}^{d} ; \\
K_{-d+2,1,1,0}^{d} \cdot K_{2,1,1,0}^{d} & =K_{2,2,2,2}^{d} ; \\
K_{-2 d+2,1,1,0}^{d} \cdot K_{2,1,1,0}^{d} & =K_{-2 d+2,-d+2,-d+2,2}^{d} ; \\
K_{-d+2,1,1,0}^{d} \cdot K_{-2 d+2,1,1,0}^{d} \cdot K_{2,1,1,0}^{d} & =K_{0,0,0,0}^{d}=0 .
\end{aligned}
$$

For $\lambda \in\{d-1,2 d-1,-1\}$, we denote by $V(\lambda)$ the eigenspace of $K_{d}$ associated with the eigenvalue $\lambda$. One computes:

\section{Lemma A.4.}

$$
\operatorname{dim} V(2 d-1)=1 ; \quad \operatorname{dim} V(-1)=(d-1)^{2} ; \quad \operatorname{dim} V(d-1)=2(d-1) .
$$

Proof. The first two results are a (quite long) direct computation. One deduces the third one using the fact that $K_{d}$ is diagonalizable (Lemma A.3).

Remark A.5. Since $K_{\lambda, 1,1,0}^{d}=K_{d}-(1-\lambda) I_{d}$, the matrix $K_{\lambda, 1,1,0}^{d}$ is invertible when $1-\lambda$ is not an eigenvalue of $K_{d}$. By Lemma A.3 this is $\lambda \notin\{-d+2,-2 d+2,2\}$. For $\lambda=-d+2$, one has

$$
\operatorname{rk} K_{-d+2,1,1,0}^{d}=d^{2}-\operatorname{dim} V(d-1)=(d-1)^{2}+1 .
$$

\section{Appendix B. Results on Kummer surfaces}

We recall some classical facts from Ino76, P̌̌Š 71 , SI77, SM74. If $S$ is a $K 3$ surface with Picard number 20 , we denote by $T_{S}$ the transcendental lattice and by $Q_{S}$ the intersection matrix of $T_{S}$ with respect to an oriented basis. Let $\mathcal{Q}$ be the set of positive definite, even integral $2 \times 2$ matrices. The class $\left[Q_{S}\right] \in \mathcal{Q} / \mathrm{SL}_{2}(\mathbb{Z})$ is uniquely determined by $S$ and $\operatorname{det} \mathrm{NS}(S)=-\operatorname{det} Q_{S}$.

For $S_{\phi}$, let $\sigma$ be the involution $(x: y: z: t) \mapsto(x: y:-z:-t)$. Then the minimal resolution of $S_{\phi} / \sigma$ is isomorphic to the Kummer surface $Y:=\operatorname{Km}\left(E_{\phi} \times E_{\phi}\right)$ and

$$
Q_{S_{\phi}}=2 Q_{Y}=4 Q_{A},
$$


where $A:=E_{\phi} \times E_{\phi}$ and $Q_{A}$ is the binary quadratic form associated with $A$ as in SM74.

For $\lambda=\frac{1+\mathrm{i} \sqrt{3}}{2}$, the group of automorphisms of the elliptic curve $E_{\phi}$ fixing a point has order 6 (since $j(\lambda)=0$ ), so $E_{\phi} \cong C_{\tau}:=\mathbb{C} / \mathbb{Z}+\tau \mathbb{Z}$ with $\tau=\frac{-1+\mathrm{i} \sqrt{3}}{2}$. By the construction of SM74, for $A=C_{\tau} \times C_{\tau}$, one has $Q_{A}=\left(\begin{array}{ll}2 & 1 \\ 1 & 2\end{array}\right)$, so $Q_{S_{\phi}}=\left(\begin{array}{ll}8 & 4 \\ 4 & 8\end{array}\right)$ and $\operatorname{det} \operatorname{NS}\left(S_{\phi}\right)=-\operatorname{det} Q_{S_{\phi}}=-48$. Moreover, observe that for $A^{\prime}=C_{\tau} \times C_{\tau^{\prime}}$ with $\tau^{\prime}=\mathrm{i} \sqrt{3}$, one has $Q_{A^{\prime}}=\left(\begin{array}{ll}4 & 2 \\ 2 & 4\end{array}\right)$, so $S_{\phi} \cong \mathrm{Km}\left(A^{\prime}\right)$.

Remark B.1. The same method has been used to compute the determinant of the Néron-Severi group of the Fermat quartic.

\section{ACKNOWLEDGEMENT}

We thank the referee for helpful suggestions and comments.

\section{REFERENCES}

[BS07] Samuel Boissière and Alessandra Sarti, Counting lines on surfaces, Ann. Sc. Norm. Super. Pisa, Cl. Sci. 6 (2007), 39-52. MR2341513

[Ino76] Hiroshi Inose, On certain Kummer surfaces which can be realized as non-singular quartic surfaces in $P^{3}$, J. Fac. Sci. Univ. Tokyo Sect. IA Math. 23 (1976), no. 3, 545-560. MR0429915 (55:2924)

[Kuw95] Masato Kuwata, Elliptic fibrations on quartic K3 surfaces with large Picard numbers, Pacific J. Math. 171 (1995), no. 1, 231-243. MR1362985 (96j:14026)

[Ogu] Keiji Oguiso, Picard numbers in a family of hyperkähler manifolds - A supplement to the article of $R$. Borcherds, L. Katzarkov, T. Pantev, N. I. Shepherd-Barron, arXiv: math. AG/0011258.

[PŠŠ71] I. I. Pjateckiı̌-Šapiro and I. R. Šafarevič, Torelli's theorem for algebraic surfaces of type K3, Izv. Akad. Nauk SSSR Ser. Mat. 35 (1971), 530-572. MR0284440 (44:1666)

[Sas68] Nobuo Sasakura, On some results on the Picard numbers of certain algebraic surfaces, J. Math. Soc. Japan 20 (1968), 297-321. MR0228495 (37:4075)

[Seg47] Beniamino Segre, On arithmetical properties of quartic surfaces, Proc. London Math. Soc. (2) 49 (1947), 353-395. MR0021952 (9:135b)

[Shi81] Tetsuji Shioda, On the Picard number of a complex projective variety, Ann. Sci. École Norm. Sup. (4) 14 (1981), no. 3, 303-321. MR644520(83i:14005)

[SI77] T. Shioda and H. Inose, On singular K3 surfaces, Complex analysis and algebraic geometry, Iwanami Shoten, Tokyo, 1977, pp. 119-136. MR0441982 (56:371)

[SM74] Tetsuji Shioda and Naoki Mitani, Singular abelian surfaces and binary quadratic forms, Classification of algebraic varieties and compact complex manifolds, Lecture Notes in Math., Vol. 412, Springer, Berlin, 1974, pp. 259-287. MR0382289 (52:3174)

[Sil94] Joseph H. Silverman, Advanced topics in the arithmetic of elliptic curves, Graduate Texts in Mathematics, vol. 151, Springer-Verlag, New York, 1994. MR1312368 (96b:11074)

Laboratoire J.A. Dieudonné UMR CNRS 6621, Université de Nice Sophia-Antipolis, Parc Valrose, 06108 Nice, France

E-mail address: samuel.boissiere@math.unice.fr

Johannes Gutenberg Universität Mainz, Institut für Mathematik, 55099 Mainz, GerMANY

E-mail address: sarti@mathematik.uni-mainz.de 